\title{
器 \\ Dopplerfluxometria das artérias intra-renais em cutias (Dasyprocta prymnolopha, Wagler 1831)
}

[Doppler flowmetry of agouti's intra-kidney arteries (Dasyprocta prymnolopha, Wagler 1831)]

\section{"Artigo Científico/Scientific Article"}

\author{
Artur da Nóbrega Carreiro ${ }^{1}$, Edijanio Galdino Silva ${ }^{1}$, Brunna Muniz Rodrigues Falcão ${ }^{1}$, Joyce \\ Galvão Souza ${ }^{1}$, João Augusto Rodrigues Alves Diniz ${ }^{1}$, Débora Vitória Fernandes Araújoº ${ }^{2}$, Marta \\ Silva Muniz ${ }^{2}$, Carlos Enrique Peña Alfaro ${ }^{1,2}$, Danilo José Ayres Menezes ${ }^{1,3 *}$
}

\author{
${ }^{1}$ Programa de Pós-Graduação em Medicina Veterinária, Universidade Federal de Campina Grande - UFCG, Patos-PB, Brasil. \\ ${ }^{2}$ Curso de Graduação em Medicina Veterinária, Universidade Federal de Campina Grande - UFCG, Patos-PB, Brasil. \\ ${ }^{3}$ Universidade Federal do Rio Grande do Norte - UFRN, Natal-RN, Brasil. \\ *Autor para correspondência/Corresponding author: E-mail: mdanayres@ gmail.com
}

\section{Resumo}

A técnica de dopplerfluxometria é importante para aferir a hemodinâmica renal, auxiliando no diagnóstico e prognóstico de problemas renais, todavia estudos com animais silvestres ainda são escassos. Assim sendo, é de extrema importância o desenvolvimento de estudos com o intuito de estabelecer valores de referências normais nas diferentes espécies, uma vez que esses dados servirão como base para a compreensão da anatomia e fisiologia, bem como, diagnóstico de doenças na clínica veterinária. Neste sentido, este trabalho teve como objetivo obter dados dos aspectos ultrassonográficos renais normais em cutias (Dasyprocta prymnolopha, Wagler 1831). Para avaliação da morfometria ultrassonográfica utilizou-se oito cutias, nas quais foram avaliados o comprimento e largura dos rins direito e esquerdo. Para avaliação da dopplerfluxometria foram utilizadas 11 cutias, sendo analisadas as velocidades do pico sistólico máximo (VPS) e diastólico final (VDF) do fluxo sanguíneo $(\mathrm{cm} / \mathrm{s})$ e do índice de resistividade (IR) das artérias arqueadas dos rins direito e esquerdo. Os valores médios do comprimento e largura em centímetros do rim direito e esquerdo variaram entre 3,05 \pm 0,13 a $1,84 \pm 0,12$ e $3,04 \pm 0,19$ a $1,91 \pm 0,10$, respectivamente. $O$ valor médio observado para o índice de resistividade na artéria arqueada dos rins direito e esquerdo foram $0,41 \pm 0,06$ e $0,39 \pm 0,05 \mathrm{~cm} / \mathrm{s}$, respectivamente. $\mathrm{O}$ índice de resistividade foi inferior aos observados em outras espécies de animais domésticos e até mesmo de humanos.

Palavras-chave: ultrassonografia renal; perfusão renal; índice de resistividade.

\begin{abstract}
The doppler flowmetry technique is important for measuring renal hemodynamics, helping in the diagnosis and prognosis of kidney problems, but there are few studies on wild animals. Thus, it is very important to develop studies to establish normal reference values in different species, because these data will serve as a base for understanding anatomy, physiology and disease diagnosis in the veterinary clinic. The objective of the present study was to obtain data on the normal kidney ultrasound aspects in agouti (Dasyprocta prymnolopha, Wagler 1831). Eight animals were used to evaluate normal morphology by ultrasound, where were assessed the length and width of the right and left kidneys. Eleven agouti were used to assess the doppler flowmetry, and the speeds of the maximum systolic peak (MSP) and final diastolic peak (FDP) of the blood flow $(\mathrm{cm} / \mathrm{s})$ and the resistivity index (RI) of the intra-kidney arteries of the right and left kidneys. The mean values of the length and width in centimeters ranged from $3.05 \pm 0.13$ to $1.84 \pm 0.12$ and $3.04 \pm 0.19$ to $1.91 \pm$ 0.10 , respectively. The mean value observed for the resistivity index in the arched arteryin of the right and left kidneys was $0.41 \pm 0.06$ and $0.39 \pm 0.05 \mathrm{~cm} / \mathrm{s}$, respectively. The resistivity index was lower than that observed in other animal species and even in humans.
\end{abstract}

Keywords: kidney ultrasound; renal perfusion; resistivity index. 


\section{Introdução}

A cutia (Dasyprocta spp.) é um roedor de porte mediano, encontrado em todo território brasileiro, com peso corporal médio de $4,53 \mathrm{~kg}$ em fêmeas e 4,37 kg nos machos, podendo chegar até $7 \mathrm{~kg}$. Alimentam-se de folhas, raízes, flores, fungos, sementes e frutos caídos (Cubas et al., 2014).

A redução na natureza e até mesmo a extinção de espécies de animais silvestres tem sido observada devido à caça indiscriminada, ao tráfico de animais e à destruição do habitat para utilização na agropecuária (Menezes et al., 2003). Neste sentido a criação de cutias em cativeiro vem como alternativa para a manutenção destes animais na natureza, como também permite a realização de estudos que envolva a espécie para o entendimento da sua biologia, possibilitando melhorias de manejo e o aprimoramento de técnicas de diagnóstico e tratamentos clínicos.

Dentre os diversos aspectos da morfofisiologia da cutia ainda não esclarecidos, $o$ sistema urinário mostra-se em destaque pela importância na manutenção da homeostasia do corpo do animal, possuindo funções metabólicas importantes, estando envolvidos na manutenção dos componentes dos líquidos corporais dentro dos valores fisiológicos e funções endócrinas (König e Liebich, 2016). Por outro lado, por serem órgãos de filtração, os rins podem ser acometidos por diversos fatores intrínsecos e extrínsecos ao organismo do animal.

Diante disso, diversos métodos são pesquisados para avaliação da função renal com a finalidade de identificar precocemente ou acompanhar as doenças renais. $\mathrm{Na}$ medicina humana, os estudos fundamentados no Doppler têm demonstrado serem eficazes para fornecer dados adicionais aos pacientes acometidos por insuficiência renal aguda, nas obstruções do trato urinário, nas neoplasias renais e em pacientes póstransplantes (Cerri et al., 1996).

Desta forma a dopplerfluxometria é uma técnica não invasiva, importante para aferir a hemodinâmica renal (Miyamoto et al., 1997) e, associada ao ultrassom convencional permite a avaliação da morfologia renal, bem como estimar o fluxo sanguíneo (Cerri et al., 1996), que são importantes para medir a capacidade de perfusão dos rins (Rivers et al., 1997a). Desta forma a dopplerfluxometria associada a estudos de imagens bidimensionais permite a avaliação da hemodinâmica renal e da morfologia renal.
Neste sentido, é de extrema importância o desenvolvimento de estudos com o intuito de estabelecer valores de referências normais nas diferentes espécies, uma vez que esses dados servirão como base para a compreensão da anatomia e fisiologia, bem como, diagnóstico de doenças na clínica veterinária. Diante disso, este estudo teve como objetivo obter dados dos aspectos morfológicos, topográficos, morfométricos e de ecogenicidade de ultrassonografias renais em cutias normais.

\section{Material e Métodos}

Foram utilizadas 11 cutias fêmeas hígidas da espécie Dasyprocta prymnolopha, provenientes do Núcleo Estudos e Preservação de Animais Silvestres (NEPAS) localizado na Universidade Federal do Piauí, Teresina, Piauí, Brasil. O experimento foi conduzido no Laboratório de Pesquisas Morfológicas da Universidade Federal de Campina Grande, Patos, Paraíba, Brasil.

Os animais foram encaminhados ao Laboratório de Pesquisas Morfológicas da Universidade Federal de Campina Grande, sendo previamente ambientados e condicionados à manipulação. Os animais foram considerados normais, após a avaliação clínica e laboratorial através de hemograma. $\mathrm{Na}$ sequência foram submetidos a tricotomias bilaterais da região abdominal lateral e um jejum alimentar de 6 horas. Durante o procedimento os animais foram contidos fisicamente e colocados em decúbito lateral, em seguida foram submetidos aos exames ultrassonográficos.

As avaliações da morfometria ultrassonográfica foram realizadas em oito cutias, com probe linear de 5 a $8 \mathrm{MHz}$, em corte longitudinal. Foram avaliadas as seguintes variáveis: comprimento e largura dos rins direito e esquerdo.

A perfusão renal foi avaliada em 11 animais por meio da técnica de ultrassonografia Doppler colorida, ajustada a janela doppler em $4 \mathrm{~mm}$. Para realização do exame utilizou-se o aparelho de ultrassom modelo Z5 Vet (Mindray ${ }^{\circledR}$, China), com probe linear de 5 a $8 \mathrm{MHz}$, sendo realizada de ambos os rins, o ângulo de insonação foi ajustado para o sentido do vaso sem ultrapassar o valor de $60^{\circ}$.

Os espectros da onda de cada artéria arqueada de ambos os rins foram obtidos e selecionados aqueles que possuíam três ondas 
consecutivas de qualidade, sem interferência de artefatos. O pico de velocidade sistólica e a velocidade diastólica final foram identificados em cada onda do espectro resultando em três valores de IR (índice de resistividade) calculados pelo aparelho para cada artéria. A partir destes três valores foram obtidas as médias e medianas do IR para cada artéria avaliada.

Os dados dos rins direito e esquerdo foram submetidos ao teste de Shapiro-Wilk, quando apresentou normalidade utilizou-se o teste t, quando não apresentava o teste $\mathrm{U}$ de MannWhitney a 5\% de significância, utilizando o programa estatístico Bioestat 5.0.

\section{Resultados}

Os rins apresentaram-se em formato de feijão em um plano longitudinal, encontrando-se situado caudal à última costela nas regiões abdominais laterais direita e esquerda, ou seja, na região dos flancos, paralelo à coluna vertebral. Os rins apresentaram o polo cranial afilado quando comparados ao polo caudal, uma cápsula renal ecóica e fina, o córtex renal hiperecóico quando comparado com a região medular e o seio medular hiperecóico. O valor médio do comprimento em centímetros do rim direito foi de $3,05 \pm 0,13$ e rim esquerdo de $3,04 \pm 0,19$. O valor médio da largura em centímetros do rim direito foi de $1,84 \pm 0,12 \mathrm{e}$ rim esquerdo de $1,91 \pm 0,10$. Após a avaliação estatística, constatou-se que não houve diferenças significativas entre o comprimento dos rins direito e esquerdo, nem na largura ( $p>0,05)$. Ou seja, os rins direito e esquerdo apresentaram-se com comprimento e largura simétricos.

A Tabela 1 mostra os valores das velocidades do pico sistólico máximo (VPS) e diastólico final (VDF) do fluxo sanguíneo ( $\mathrm{cm} / \mathrm{s})$ e do índice de resistividade (IR) das artérias intrarenais dos rins direito e esquerdo. $\mathrm{O}$ valor médio observado para o pico sistólico máximo no rim direito foi de $32,42 \pm 3,34 \mathrm{~cm} / \mathrm{s}$ e no esquerdo de $34,10 \pm 1,37 \mathrm{~cm} / \mathrm{s}$. O valor médio observado para o pico diastólico final no rim direito foi de 19,15 \pm $2,48 \mathrm{~cm} / \mathrm{s}$ e no rim esquerdo $20,87 \pm 1,92 \mathrm{~cm} / \mathrm{s}$. O valor médio observado para o índice de resistividade na artéria arqueada do rim direito foi de $0,41 \pm 0,06 \mathrm{~cm} / \mathrm{s}$ e no rim esquerdo de $0,39 \pm 0,05$ $\mathrm{cm} / \mathrm{s}$. Após a análise estatística não foram observadas diferenças entre o rim direito e esquerdo quanto à comparação dos valores da VPS, VDF e IR ( $p>0,05)$.

\section{Discussão}

A ultrassonografia é uma técnica em ascensão na clínica veterinária de animais silvestres, sendo este o primeiro estudo da utilização de ultrassonografia para avaliação renal em cutias criadas em cativeiros. Com isso, esperase que esses resultados sirvam como base para auxiliar os médicos veterinários no diagnóstico de doenças renais nessa espécie.

Os parâmetros ultrassonográficos e a morfometria renal assemelharam-se às descritas na literatura em cães e gatos (Nyland et al., 2005), assim como em lobo guará (Guimarães et al., 2013). Os rins direito e esquerdo são praticamente do mesmo tamanho e formato.

A utilização da técnica de ultrassonografia Doppler é um método atual destinado ao auxílio de diagnóstico na medicina humana e veterinária. $\mathrm{Na}$ Medicina Veterinária encontram-se alguns estudos do emprego desta técnica, principalmente envolvendo cães e gatos (Rivers et al., 1997a).

Em roedores silvestres os estudos de ultrassonografia estão restritos a poucos trabalhos, como ultrassonografia doppler da placenta, vasos maternos e fetais durante a gestação em cutia (Dasyprocta prymnolopha) (Sousa et al., 2016) e em pacas (Agouti paca) (Oliveira et al., 2003). Outros trabalhos envolvendo roedores foram realizados em ratos (Fisch et al., 2016). No mais, até o momento há uma escassez de estudos com animais silvestres envolvendo a utilização de ultrassonografia, principalmente a dopplerfluxometria.

Na literatura, já foi constatado que o IR pode servir como indicativo para o diagnóstico de patologias renais. Um estudo realizado com gatos constatou diferenças no IR entre gatos saudáveis e com doenças renais (Tipisca et al., 2015).

Os autores observaram que em gatos saudáveis o IR médio no rim direito foi $0,54 \pm 0,07$ e no rim esquerdo $0,59 \pm 0,08$. Observaram ainda que, em gatos doentes no rim esquerdo, o IR em doença renal crônica foi de $0,73 \pm 0,12$ e na lesão renal aguda $0,72 \pm 0,08$ e para o rim direito, o IR com doença renal crônica $0,72 \pm 0,11$, na lesão renal aguda de $0,74 \pm 0,08$, em doença renal policística $0,77 \pm 0,11$ e no tumor renal $0,74 \pm$ 0,001 . Dessa forma, observa-se elevação do IR frente a essas alterações renais nos gatos, os quais, quando saudáveis, apresentaram valores semelhantes aos observados nas cutias, fornecendo um padrão de comportamento do IR para identificar essas lesões em cutias. 
Tabela 1. Valores das velocidades do pico sistólico máximo (VPS) e diastólico final (VDF) do fluxo sanguíneo $(\mathrm{cm} / \mathrm{s})$ e do índice de resistividade (IR) das artérias arqueadas dos rins direito e esquerdo em cutias (Dasyprocta prymolopha) criadas em cativeiro.

\begin{tabular}{ccccccc}
\hline \multirow{2}{*}{ Animal } & \multicolumn{3}{c}{ Rim Direito } & \multicolumn{3}{c}{ Rim Esquerdo } \\
\cline { 2 - 7 } & VPS & VDF & IR & VPS & VDF & IR \\
\hline $\mathbf{1}$ & 31,26 & 18,66 & 0,40 & 34,40 & 20,46 & 0,41 \\
$\mathbf{2}$ & 29,23 & 17,76 & 0,39 & 36,20 & 23,61 & 0,35 \\
$\mathbf{3}$ & 29,46 & 18,66 & 0,37 & 35,53 & 27,21 & 0,23 \\
$\mathbf{4}$ & 31,26 & 17,31 & 0,45 & 36,20 & 20,69 & 0,43 \\
$\mathbf{5}$ & 30,13 & 17,54 & 0,42 & 33,06 & 20,46 & 0,38 \\
$\mathbf{6}$ & 33,28 & 19,11 & 0,43 & 34,40 & 21,59 & 0,37 \\
$\mathbf{7}$ & 33,06 & 20,91 & 0,37 & 33,51 & 20,01 & 0,40 \\
$\mathbf{8}$ & 34,85 & 19,34 & 0,45 & 33,06 & 18,21 & 0,45 \\
$\mathbf{9}$ & 28,33 & 13,94 & 0,51 & 32,61 & 19,56 & 0,40 \\
$\mathbf{1 0}$ & 37,10 & 22,71 & 0,39 & 33,73 & 18,89 & 0,44 \\
$\mathbf{1 1}$ & 38,68 & 24,74 & 0,36 & 32,38 & 18,89 & 0,42 \\
\hline Média & $\mathbf{3 2 , 4 2} \pm \mathbf{3 , 3 4}$ & $\mathbf{1 9 , 1 5} \pm \mathbf{2 , 4 8}$ & $\mathbf{0 , 4 1} \pm \mathbf{0 , 0 6}$ & $\mathbf{3 4 , 1 0} \pm \mathbf{1 , 3 7}$ & $\mathbf{2 0 , 8 7} \pm \mathbf{1 , 9 2}$ & $\mathbf{0 , 3 9} \pm \mathbf{0 , 0 5}$ \\
\hline
\end{tabular}

Valores com letras diferentes da mesma variável diferem entre si pelo Teste t ou U de Mann-Whitney a 5\% de significância.

De forma geral, os valores de IR observados nas artérias arqueadas no nosso estudo variaram entre 0,23 e 0,51 , com valores médios variando entre $0,41 \pm 0,06$ e $0,39 \pm 0,05$, nos rins direito e esquerdo, respectivamente. Os valores do IR aqui observados foram inferiores a outros trabalhos já mencionados. Assim como em suínos que apresentaram média do IR nas artérias interlobares de 0,63 (Rawashdeh et al., 2000). Entretanto em um estudo com girafas o IR médio da artéria renal foi de 0,26 (Damkjær et al., 2015), inferior aos valores observados nas artérias arqueadas em cutias.

Os valores normais propostos para IR renal em cães normais nas artérias intra-renais é 0,73 , em gatos normais de 0,71 (Rivers et al., 1997a) e em humanos é 0,70 (Platt, 1992). Além disso, o valor médio do IR das artérias arqueadas dos rins direito $0,41 \pm 0,06$ e esquerdo $0,39 \pm 0,05$ das cutias foram menores que os encontrados em outros animais normais, cuja variação encontra-se 0,60 e 0,62 (Morrow et al., 1996).

Os valores de IR observados nesse estudo foram próximos aos valores encontrados em cães sedados (Rivers et al., 1997b), já em gatos sedados observou-se valores superiores a 0,52 (Rivers et al., 1996), acima do valor superior encontrado em nosso trabalho. Em macacos-cinomolgo (Macaca fascicularis) sedados, observou-se valores médios de IR de 0,54 (Gaschen et al., 2000), superior à média observada em nosso estudo. Entretanto os agentes anestésicos podem alterar a hemodinâmica renal (Mitchell et al., 1998). Diante disso, esses dados mostram a importância do estabelecimento de valores de referência por espécie. Portanto, esse é o primeiro estudo que descreve os valores das velocidades sistólica máxima e diastólica mínima dos fluxos sanguíneos nas artérias arqueadas em cutias.

Diante disso, espera-se que esses valores sirvam como parâmetros para futuras comparações com estudos realizados com a mesma espécie com animais acordados e até mesmo com espécies diferentes.

\section{Conclusão}

A técnica de dopplerfuxometria em cutias condicionadas é relativamente fácil de realizar. Determinamos os valores do fluxo sanguíneo renal das artérias-arqueadas em cutias. Consideramos que esses resultados podem contribuir para uma melhor compreensão da ultrassonografia renal nesta espécie.

\section{Conflito de Interesse}

Os autores declaram não existir conflito de interesse.

\section{Comitê de Ética}

Os protocolos metodológicos deste projeto foram aprovados pelo Ministério do Meio Ambiente, por meio do Sistema de Autorização e Informação da Biodiversidade - SISBIO, do Instituto Chico Mendes - ICMBio, sob protocolos No 45046-1 e 47944-1, e pelo Comitê de Ética no Uso de Animais da UFCG, CEP No 237 - 2014. 


\section{Referências}

Cerri, G.G.; Molnar, L.; Vezozzo, D.C. Doppler. São Paulo: Sarvier, 1996. 291p.

Cubas, Z.S.; Silva, J.C.R.; Catão-Dias, J.L. Tratado de animais selvagens: medicina veterinária. $2^{\mathrm{a}}$ ed. São Paulo: Roca, 2014. $2512 \mathrm{p}$.

Damkjær, M.; Wang, T.; Brøndum, E.; Østergaard, K.H.; Baandrup, U.; Hørlyck, A.; Hasenkam, J.M.; Smerup, M.; Funder, J.; Marcussen, N.; Danielsen, C.C.; Bertelsen, M.F.; Grøndahl, C.; Pedersen, M.; Agger, P.; Candy, G.; Aalkjær, C.; Bie, P. The giraffe kidney tolerates high arterial blood pressure by high renal interstitial pressure and low glomerular filtration rate. Acta Physiologica, 214(4): 497-510, 2015.

Fisch, S.; Liao, R.; Hsiao, L.L.; Lu, T. Early detection of drug-induced renal hemodynamic dysfunction using sonographic technology in rats. Journal of Visualized Experiments: JoVE, 109: 1-7, 2016.

Gaschen, L.; Menninger, K.; Schuurman, H. Ultrasonography of the normal kidney in the cynomolgus monkey (Macaca fascicularis): morphologic and Doppler findings. Journal of Medical Primatology, 29(2): 76-84, 2000.

Guimarães, L.D.; Hage, M.C.F.; Paula, T.A.; Borges, A.P, Carretta Júnior, M.; Oliveira, A.R.; Silva, V.H. Abdominal and pelvic ultrasound study of the maned wolf (Chrysocyon brachyurus). Pesquisa Veterinária Brasileira, 33(2): 265-272, 2013.

König, H.; Liebich, H.G. Anatomia dos animais domésticos: texto e atlas colorido. $6^{\text {th }}$ ed. Porto Alegre: ArtMed, 2016. 824 p.

Menezes, D.J.A.; Carvalho, M.A.M.; Assis-Neto, A.C.; Oliveira, M.F.; Farias, E.C.; Miglino, M.A.; Medeiros, G.X. Morfologia dos órgãos genitais externos do macho de cutia (Dasyprocta aguti. Linnaeus, 1766). Brazilian Journal of Veterinary Research and Animal Science, 40: 148-153, 2003.

Mitchell, S.K.; Toal, R.L.; Daniel, G.B.; Rohrbach, B.W. Evaluation of renal hemodynamics in awake and isoflurane-anesthetized cats with pulsed-wave doppler and quantitative renal scintigraphy. Veterinary Radiology \& Ultrasound, 39(5): 451-458, 1998.

Miyamoto, T.; Shirahama, M.; Kiryu, C.; Mwanza, T.; Okumura, M.; Hagio, M.; Fujinaga, T. Comparison of systemic and renal hemodynamics measured by Doppler ultrasonography in canine experimental hypovolemia. Journal of Veterinary Medical Science, 59(5): 347-352, 1997.

Morrow, K.L.; Salman, M.D.; Lappin, M.R.; Wrigley, R. Comparison of the resistive index to clinical parameters in dogs with renal disease. Veterinary Radiology \& Ultrasound, 37(3): 193-199, 1996.

Nyland, T.G.; Mattoon, J.S.; Herrsell, E.J.; Wisner, E.R. Ultra-som diagnóstico em pequenos animais. $2^{\mathrm{a}}$ ed. São Paulo: Roca, 2005. 506p.

Oliveira, F.S.; Machado, M.R.F.; Canola, J.C. Real time B-mode ultrasound in pacas pregnancy (Agouti paca, Linnaeus, 1766). Brazilian Journal of Veterinary Research and Animal Science, 40(1): 73-78, 2003.

Platt, J.F. Duplex Doppler evaluation of native kidney dysfunction: obstructive and nonobstructive disease. AJR: American Journal of Roentgenology, 158(5): 10351042, 1992.

Rawashdeh, Y.F.; Mortensen, J.; Horlyck, A.; Olsen, K.O.; Fisker, R.V.; Schroll, L.; Frokiaer, J. Resistive index: an experimental study of the normal range in the pig. Scandinavian Journal of Urology and Nephrology, 34(1): 10-14, 2000.

Rivers, B.J.; Walter, P.A.; O'Brien, T.D.; Polzin, D.J. Duplex doppler estimation of pourcelot resistive index in arcuate arteries of sedated normal cats. Journal of Veterinary Internal Medicine, 10(1): 28-33, 1996.

Rivers, B.J.; Walter, P.A.; Polzin, D.J.; King, V.L. Duplex doppler estimation of intrarenal pourcelot resistive index in dogs and cats with renal disease. Journal of Veterinary Internal Medicine, 11(4): 250-260, 1997a.

Rivers, B.J.; Walter, P.A.; Letourneau, J.G.; Finlay, D.E.; Ritenour, E.R.; King, V.L.; O’brien, T.D.; Polzin, D.J. Duplex Doppler estimation of resistive index in arcuate arteries of sedated, normal female dogs: implications for use in the diagnosis of renal failure. Journal of the American Animal Hospital Association, 33(1): 69-76, $1997 \mathrm{~b}$.

Sousa, F.C.A.; Pessoa, G.T.; Moura, L.S.; Rodrigues, R.P.S.; Diniz, A.N.; Souza, A.B.; Silva, E.G.; Sanches, M.P.; Silva-Filho, O.F.; Guerra, P.C.; Sousa, J.M.; Neves, W.C.; Alves, F.R. Doppler ultrasound of the placenta and maternal and fetal vessels during normal 
gestation in captive agoutis (Dasyprocta prymnolopha, Wagler, 1831). Theriogenology, 86(8): 1921-1930, 2016.

Tipisca, V.; Murino, C.; Cortese, L.; Mennonna,

G.; Auletta, L.; Vulpe, V.; Meomartino, L.
Resistive index for kidney evaluation in normal and diseased cats. Journal of Feline Medicine and Surgery, 18(6): 471-475, 2015. 